\title{
Experimental study on atomization characteristics of two common spiral channel pressure nozzles
}

\author{
Han Han ${ }^{3}$, Pengfei Wang 1,2, *, Ronghua Liu ${ }^{1,3}$, Yongjun $\mathrm{Li}^{3}$, Jan Wang ${ }^{3}$, and Yidan \\ Jiang ${ }^{3}$ \\ ${ }^{1}$ Work Safety Key Lab on Prevention and Control of Gas and Roof Disasters for Southern Coal Mines, \\ Hunan University of Science \& Technology, Xiangtan Hunan 411201, China \\ ${ }^{2}$ Hunan Province Key Laboratory of Coal Resources Clean-utilization and Mine Environment \\ Protection, Hunan University of Science \& Technology, Xiangtan Hunan 411201, China \\ ${ }^{3}$ School of Resource, Environment \& Safety Engineering, Hunan University of Science \& \\ Technology, Xiangtan 411201, China
}

\begin{abstract}
Spiral channel pressure nozzles are commonly used pressure nozzles in practical workplaces. In this paper, two kinds of spiral channel type pressure nozzles, namely, spiral hole type and spiral non-porous type, the atomization characteristics and dust reduction efficiency under different spray pressures are discussed and compared. Based on the experimental method, based on the self-designed spray dust-reducing roadway experimental platform, the macro-atomization characteristics of the two nozzles, namely the flow rate, the atomization angle, the range, and the droplet size, were measured. The following conclusions were drawn: (1) The flow rates of both nozzles increase with increasing spray pressure, and the flow coefficient of the spiral non-porous nozzle is small. (2) The change of the atomization angle of the two nozzles first increases and then decreases with the increase of the spray pressure, and the atomization angle of the spiral non-porous nozzle is larger. At the same time, the range of the two nozzles gradually increases as the spray pressure increases, and the range of the spiral perforated nozzle is always larger than that of the spiral non-porous nozzle. (3) When the spray pressure is gradually increased, the droplet size of the two nozzles selected in the experiment is gradually reduced, and the droplet size of the spiral perforated nozzle is always larger than that of the spiral non-porous nozzle before $5 \mathrm{MPa}$, and then gradually Become smaller. The main reason why the droplet size decreases with the increase of the spray pressure is that the increase of the spray pressure leads to an increase in the spray speed of the water droplets, so that the water droplets are completely split when they are ejected from the nozzle, resulting in a smaller droplet size. In summary, when the spray pressure required in the actual working environment is low, the use of a spiral non-porous nozzle is more conducive to dust reduction.
\end{abstract}

* Corresponding author: WANG Peng-fei (1984.08-), male, A.P., 13789303851 (cell).

E-mail address: pfwang666666@126.com 


\section{Introduction}

With the rapid development of China's social economy and the continuous advancement of science and technology, mechanization has covered all aspects of people's production and life. Taking coal mining as an example, the degree of mechanization is deepening, which will increase the intensity of coal mining, which is accompanied by a sharp increase in the dust of coal mine working, which seriously affects the safe production of mines and the health of workers. The problem of coal dust has gradually become an important factor restricting the sustainable development of coal mines, and has become an influential factor to ensure the safe production of coal mines. Excessive concentration of coal dust will affect the working environment in the underground, speed up the wear and tear of machine instruments, and more serious will threaten the health of workers. According to the National Occupational Disease Report issued by the National Health and Health Commission of China, a total of 27,420 occupational diseases were reported in 2017. Among them, 24,206 pneumoconiosis cases accounted for $88.29 \%$ of the total number of reports. Therefore, it is extremely urgent to adopt effective dust control measures to reduce the dust concentration in production sites ${ }^{[1-9]}$. At present, the main dust prevention measures adopted at the mining face at home and abroad include coal pre-filling water, ventilation and dust control, dust collector dust purification, closed dust extraction and personal protection. Among them, spray dust reduction is economical, simple and practical. It will be widely used as the main means of dust reduction for a long time ${ }^{[10-11]}$.

With the development of science and technology, the research and analysis of spray dust reduction theory has generally gone through three stages. The first stage was carried out due to the emergence and promotion of cyclone dust collectors. Due to the increasing demand for environmental protection, cyclones are widely used for gas dedusting. At this time, the research on the principle of wet spray dusting still stays in the rational analysis based on experience. People can only judge from experience how much spray pressure and spray flow or what type of nozzle can get the best dust removal effect. Little is known about the microscopic dust removal mechanism. With the introduction of the theory of tiny water mist capture in 1976, Skowinger and Brown proposed that the research on spray dust reduction began to be qualitative and macroscopically turned to quantitative and microscopic. This is the second stage of the development of spray dust reduction theory. The third stage is accompanied by the rapid development of various physical and chemical dust removal technologies. At this stage, people have made a perfect analysis of the theory of spray dust reduction: the main principle of dust collection and trapping in air is to rely on the inertial collision, interception, gravity, electrostatic force, eddy condensation and other effects of the droplets. The study of dustfall is going deep and comprehensive ${ }^{[12-13]}$.

On the basis of theoretical research on atomization mechanism, many scholars at home and abroad apply new methods, and carry out deeper research on spray dust reduction through new perspectives, thus providing better theoretical guidance for spray dust reduction in coal mines. . For example, Ma Suping ${ }^{[14-15]}$ studied the floating dust in the return airway of coal mines as the research object, deeply studied the mechanism of spray dust reduction, analyzed the factors affecting the dust deposition efficiency, established the corresponding mathematical model, and used the MATLAB software to draw dust. The sedimentation efficiency curve is analyzed, and the smaller the water mist particle size is, the higher the dust removal efficiency is, but the dust particles of different particle sizes correspond to a better water mist particle size. Wang Pengfei et al ${ }^{[5]}$ used the dust spray test system designed by himself to apply the dust mass concentration tester to measure the dust reduction effect of the spiral pressure nozzle commonly used in underground under 
different conditions, and found that the nozzle is the most under the same spray pressure. Good diameter and relationship between spray pressure, roadway wind speed, airflow dust mass concentration and dust reduction efficiency. Hadi Taghavifar et al. ${ }^{[16]}$ studied the influence of nozzle dip angle on the flow characteristics of the nozzle, and based on the comparison between simulation and experiment, the simulation based on the prediction of the effectiveness of the spray structure in terms of penetration and droplet diameter. Related laws. Guan Xiaoqiong et al. ${ }^{[17]}$ studied the relationship between the spray amount of the spiral nozzle and the spray pressure, and the particle size distribution characteristics of the atomized droplets. The experimental results show that with the increase of spray pressure, the spray amount increases correspondingly and gradually becomes gentle. The average droplet size tends to be uniform with the increase of pressure, and the particle size ratio formed by atomization of large nozzle The small nozzle is slightly larger. Li Chuan et al. ${ }^{[18]}$ proposed a new type of spiral nozzle with an involute atomizing surface. The spatial geometry model of the jet profile is derived by using the dynamic coordinate system to the fixed coordinate system mapping. The designed profile is simulated by Matlab. The results verify the validity of the proposed geometric model and provide a theoretical basis for the design and manufacture of spiral nozzles.

In summary, when investigating the influencing factors of spray dust reduction and spiral channel nozzles, past studies have paid more attention to external environmental factors such as spray pressure, and have little research on the effect of nozzle internal structure on dust removal efficiency, and we are in coal mines. The internal structure of the nozzles commonly used in underground is different. Therefore, the author will use the self-designed spray dust reduction test system to study two different spiral channel nozzles commonly used in coal mines, in order to provide theoretical guidance for coal mine downhole dust reduction operations.

\section{Experimental systems and solutions}

\subsection{Nozzle selection}

Through the investigation of the actual production conditions, this paper selects two kinds of pressure nozzles commonly used in production as the experimental object. The two nozzles are spiral-type pressure nozzle and spiral non-porous pressure nozzle, as shown in Figure 1. After consulting the relevant data, it was found that in the downhole spray dust reduction, when the water consumption is not limited, in order to ensure the dust reduction efficiency of the whole dust and the respirable dust at the same time, it is preferable to select a nozzle with a diameter of $1.5 \mathrm{~mm}$, and conversely, when the water consumption is limited. When using a nozzle with a diameter of $1.2 \mathrm{~mm}$, it is advisable to use it. In order to reduce the water consumption, a nozzle with a diameter of $1.2 \mathrm{~mm}$ was selected in the experiment ${ }^{[19-20]}$. 


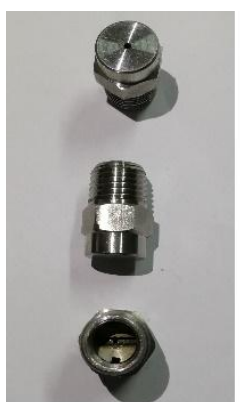

(a)

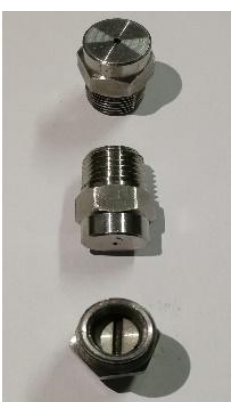

(b)

Fig. 1. Schematic diagram of nozzle structure, (a) spiral perforated nozzle, (b) spiral non-porous nozzle.

\subsection{Experimental platform}

The experiment adopts a self-designed spray dust-reducing system that can simulate the state of wind, spray and dust in the coal mining face under the coal mine, as shown in Figure 2. The self-designed spray dust reduction system mainly consists of the following parts: a roadway model, a water supply system, a dust generating system, a measuring system, etc., wherein the components of the roadway model include a mixing section, a rectifying section, a measuring section, a spray section, and a dust removing fan. And the export section; the water supply system is mainly composed of a high-pressure water pump and a water tank; the measurement system mainly includes a Malvern Spraytec laser particle size detector, a flow meter, and the like.

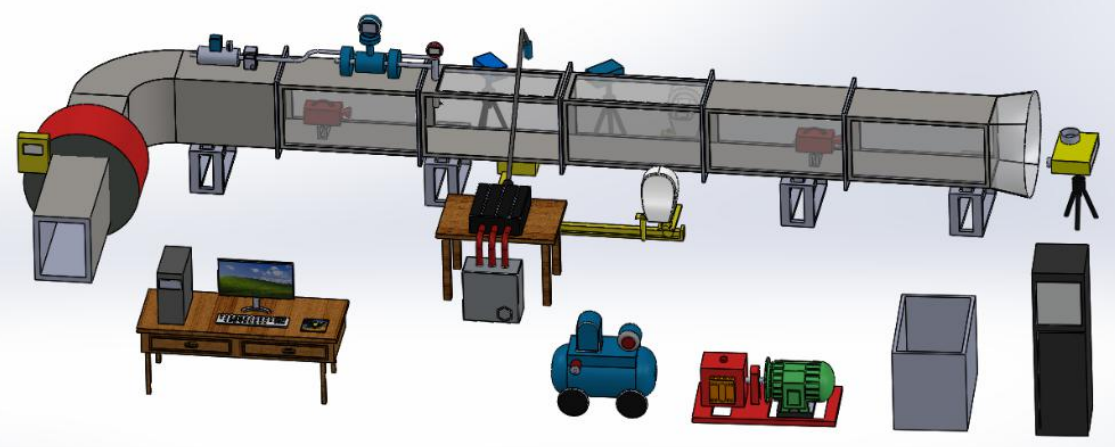

Fig. 2. Schematic diagram of the spray dust reduction experiment system. 


\section{3 atomization characteristics measuring instruments and programs}

The nozzle atomization characteristic parameters include parameters such as nozzle flow rate, atomization angle, range, and droplet size. Figure 3 shows the instruments used in the atomization characteristics experiment. In the experiment, the spray field of the nozzle was taken with a high-performance digital camera, and the atomization angle and range calculation were performed with Image-Pro Plus 6.0 post-processing software. The Malvern Droplet Particle Size Analyzer is based on the principle of line measurement. The measured droplet data is the value of the droplet size on the line through which the laser beam passes. Select the $50 \mathrm{~cm}$ vertical section center in front of the spout as the collection line for the droplet size parameter. At the same time, combined with the actual industrial production process, the spray pressure is selected in the range of $1 \sim 8 \mathrm{MPa}$ to measure the flow rate, atomization angle, range and droplet size of the four nozzles.
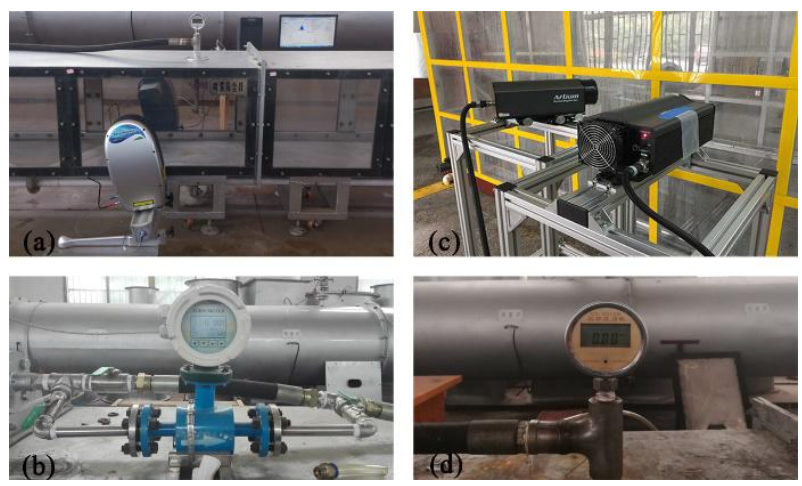

Fig. 3. atomization characteristics experimental instrument, (a) Malvern real-time particle size analysis, (b) YY-LED15K4C electromagnetic flowmeter, (c) high-speed camera, (d) DX-801XB digital pressure gauge.

\section{Experimental results and analysis}

\subsection{Macro atomization characteristic parameters}

By consulting the relevant data, the nozzle flow rate is basically satisfied with the change of the spray pressure:

$$
Q=\frac{\pi}{4} c_{q} d^{2} \sqrt{\frac{2 p}{\rho}} \times 10^{-3}
$$

Where: $Q$ is the nozzle flow rate, $\mathrm{L} / \mathrm{min} ; \mathrm{C}_{\mathrm{q}}$ is the flow coefficient, $d$ is the nozzle diameter, $\mathrm{mm} ; p$ is the spray pressure, $\mathrm{MPa} ; \rho$ is the liquid density, $\mathrm{kg} / \mathrm{L}$. The experimental data were analyzed by fitting analysis using spss statistical analysis software to obtain the flow coefficients of the two nozzles.

In the experimental system, the flow rate of the two nozzles under different pressures 
was measured by a flow meter, as shown in Table 1 .

Table 1. Relationship between flow rate $\mathrm{L}$ of two nozzles and water supply pressure (unit: $\mathrm{L} / \mathrm{min}$ )

\begin{tabular}{cccccccccc}
\hline Nozzle & 1 & 2 & 3 & 4 & 5 & 6 & 7 & 8 & Flow \\
& $\mathrm{MPa}$ & $\mathrm{MPa}$ & $\mathrm{MPa}$ & $\mathrm{MPa}$ & $\mathrm{MPa}$ & $\mathrm{MPa}$ & $\mathrm{MPa}$ & $\mathrm{MPa}$ & Coefficient \\
\hline $\begin{array}{c}\text { spiral } \\
\text { perforated } \\
\text { nozzle } \\
\text { spiral }\end{array}$ & 2.50 & 3.17 & 3.83 & 4.17 & 5.17 & 5.33 & 5.67 & 6.17 & 0.564 \\
$\begin{array}{c}\text { non-porous } \\
\text { nozzle }\end{array}$ & 1.83 & 2.33 & 2.83 & 3.17 & 3.83 & 4.17 & 4.33 & 4.83 & 0.432 \\
\hline
\end{tabular}

As can be seen from the analysis data, the flow rates of both nozzles gradually increase as the spray pressure increases. At the same time, it can be found that the flow coefficient of the spiral non-porous nozzle is small, because when the nozzle diameter and the spray pressure are fixed, the nozzle inlet fillet radius has a decisive influence on the influence of the flow coefficient thereof. In actual work, the increase of the nozzle flow rate can increase the atomization water volume per unit space. Therefore, in the actual production process, in order to effectively improve the dust reduction efficiency, a spiral perforated nozzle can be selected.

At the same time, the size of the nozzle atomization angle determines the size of the nozzle spray nozzle droplet coverage, which reflects the effective range of the nozzle dust removal, and is an important parameter of the nozzle atomization characteristics. In the experiment, the atomization angle of the nozzle was photographed by a high-speed camera, and the atomization angle taken at a spray pressure of 1 to $8 \mathrm{MPa}$ is shown in Fig. 5. After the image processing software of Image-Pro Plus 6.0, the measurement data of the atomization angle of the two nozzles selected by the experiment under different pressures were obtained.

(a)

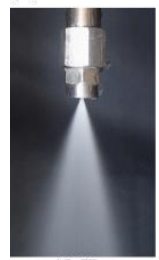

(b)

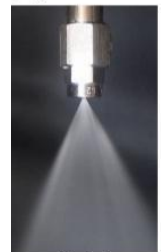

$1 \mathrm{MPa}$

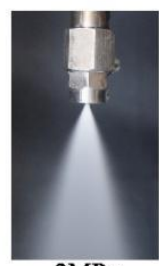

$2 \mathrm{MPa}$

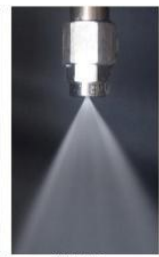

$2 \mathrm{MPa}$

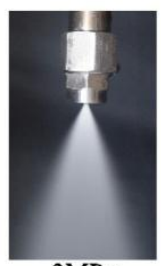

$3 \mathrm{MPa}$

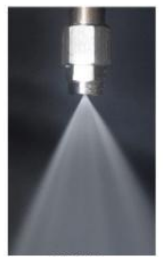

$3 \mathrm{MPa}$

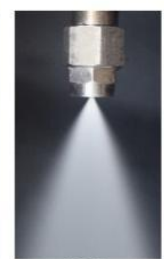

4MPa

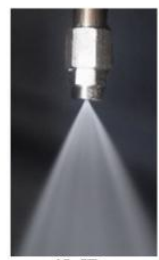

$4 \mathrm{MPa}$

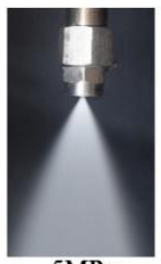

$5 \mathrm{MPa}$

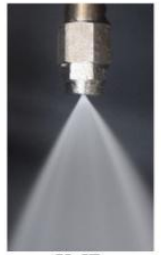

$5 \mathrm{MPa}$

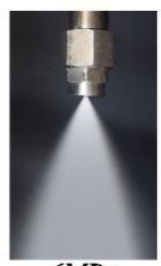

$6 \mathrm{MPa}$

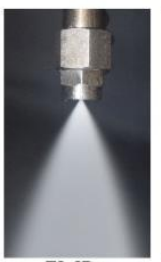

$7 \mathrm{MPa}$

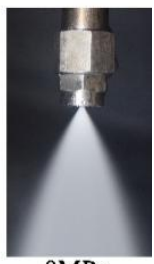

$8 \mathrm{MPa}$

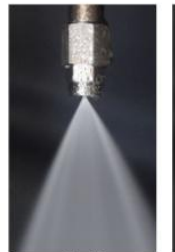

$6 \mathrm{MPa}$

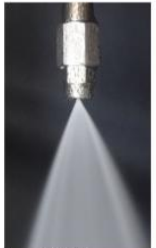

7MPa

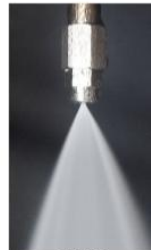

8MPa

Fig. 5. Nozzle atomization angle, (a) spiral perforated nozzle, (b) spiral non-porous nozzle. 
In order to investigate the range change of the four nozzles selected in the experiment under different pressures, we set the spray pressure to $1 \mathrm{MPa}, 2 \mathrm{MPa}, 3 \mathrm{MPa}, 4 \mathrm{MPa}, 5$ $\mathrm{MPa}, 6 \mathrm{MPa}, 7 \mathrm{MPa}, 8 \mathrm{MPa}$. The distance traveled by the water droplets of different nozzles under different spray pressures is measured by a measuring tape. For convenience of comparison, the obtained nozzle atomization angle was integrated with the experimental data of the range to obtain Fig. 6.

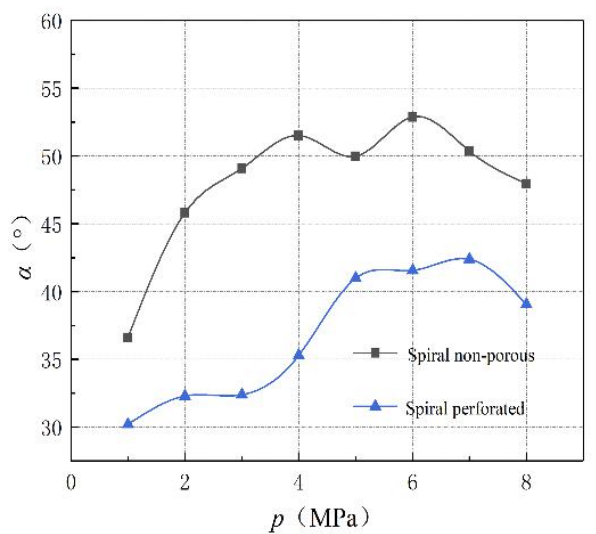

(a)

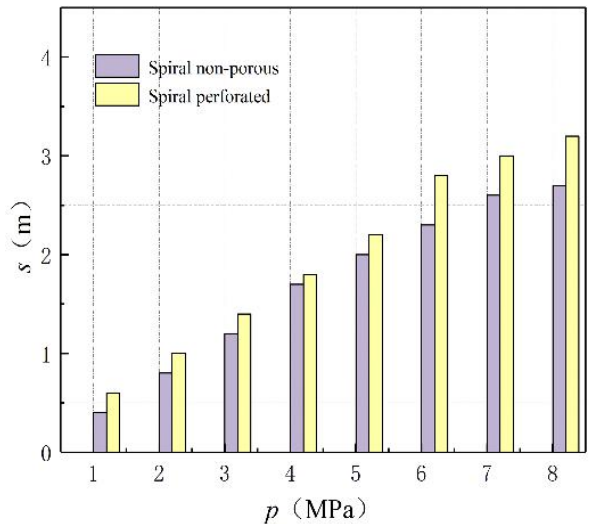

(b)

Fig. 6. Variation of spray angle and range with spray pressure, (a) atomization angle, (b) range.

Excluding the experimental error, it can be found that the change of the atomization angle of the two nozzles increases first and then decreases with the increase of the spray pressure, and the atomization angle of the spiral non-porous nozzle is larger. At the same time, the range of the two nozzles gradually increases as the spray pressure increases, and the range of the spiral perforated nozzle is always larger than that of the spiral non-porous nozzle.

This is because as the spray pressure increases, the nozzle flow rate increases, increasing the swirling force inside the nozzle, thereby increasing the radial velocity and atomization angle of the nozzle outlet spray. Since the particle size of the droplet is too small when the spray pressure is too large, the pressure inside the water column becomes small, and the atomization angle becomes small. At the same time, since there is no channel in the middle of the spiral non-porous nozzle, the water volume is concentrated outside the atomization angle, and the atomization angle is hollow, resulting in a large atomization angle. The spiral perforated nozzle has a larger radial velocity due to its internal swirling force, which results in a larger radial velocity of the droplet, resulting in a longer range than the spiral non-porous nozzle. Therefore, in the working environment requiring large-area dust reduction, a spiral non-porous nozzle can be selected to reduce the installation cost. When the nozzle installation point is far away from the dust-reducing environment, the spiral-type nozzle is most suitable.

\subsection{Droplet particle size}

In the experiment, the Malvern Spraytec laser particle size detector was used to measure the droplet size under the spray pressure change. The collection point of the droplet size parameter was set at the axis position $50 \mathrm{~cm}$ in front of the nozzle to facilitate the 
experiment. With the installation of the equipment. In the experiment, $\mathrm{DV}_{[3][2]}$ and $\mathrm{DV}_{[4][3]}$ respectively represent the Sol and the weight-averaged mean diameter; $\mathrm{DV}_{10}, \mathrm{DV}_{50}$ and $\mathrm{DV}_{90}$, respectively, indicate that the particle volume smaller than this particle accounts for the total volume of all particles. $10 \%, 50 \%$.it can be found that $\mathrm{DV}_{[4][3]}$ is larger in the parameters describing the average particle size of the droplets, while $\mathrm{DV}_{50}$ and $\mathrm{DV}_{[3][2]}$ are closer. In order to facilitate data comparison, the data in the table is sorted to obtain Fig. 7.

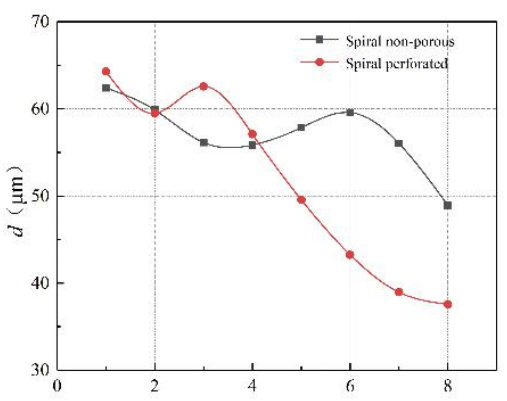

$p(\mathrm{MP})$

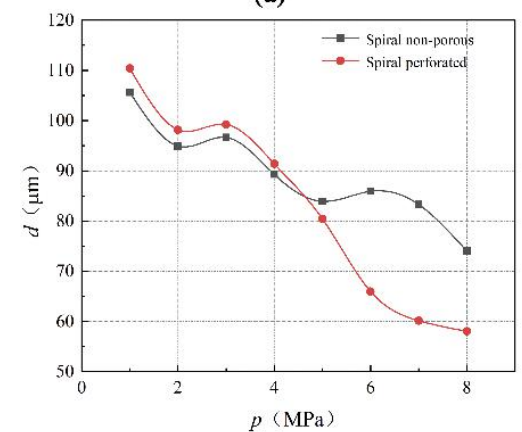

(c)

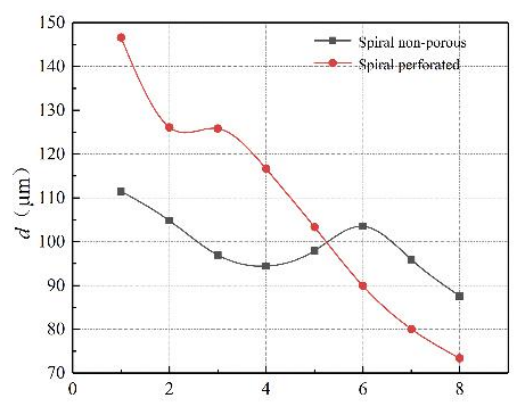

${ }^{p}(\mathrm{M})$

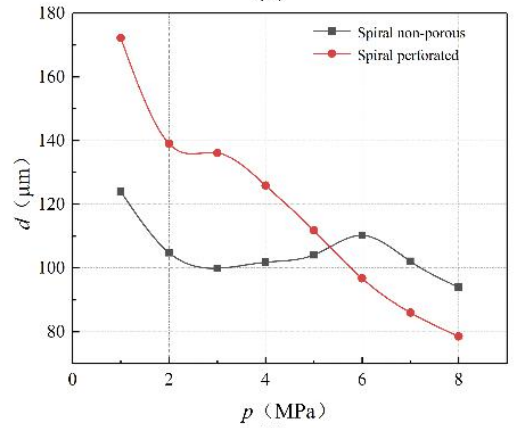

(d)

Fig. 7. Droplet droplet size as a function of pressure, (a) $\mathrm{DV}_{10}$, (b) $\mathrm{DV}_{50}$, (c) $\mathrm{DV}_{[3][2]}$, (d) $\mathrm{DV}_{[4][3] \text {. }}$.

At the same time, through the Malvern laser particle size detector, the droplet size $\left(\mathrm{DV}_{50}\right)$ distribution, volume frequency and cumulative distribution of the four nozzles under the change of spray pressure from 1 to $8 \mathrm{MPa}$ can be obtained, and the experimental data is combined with Fig. 8. 

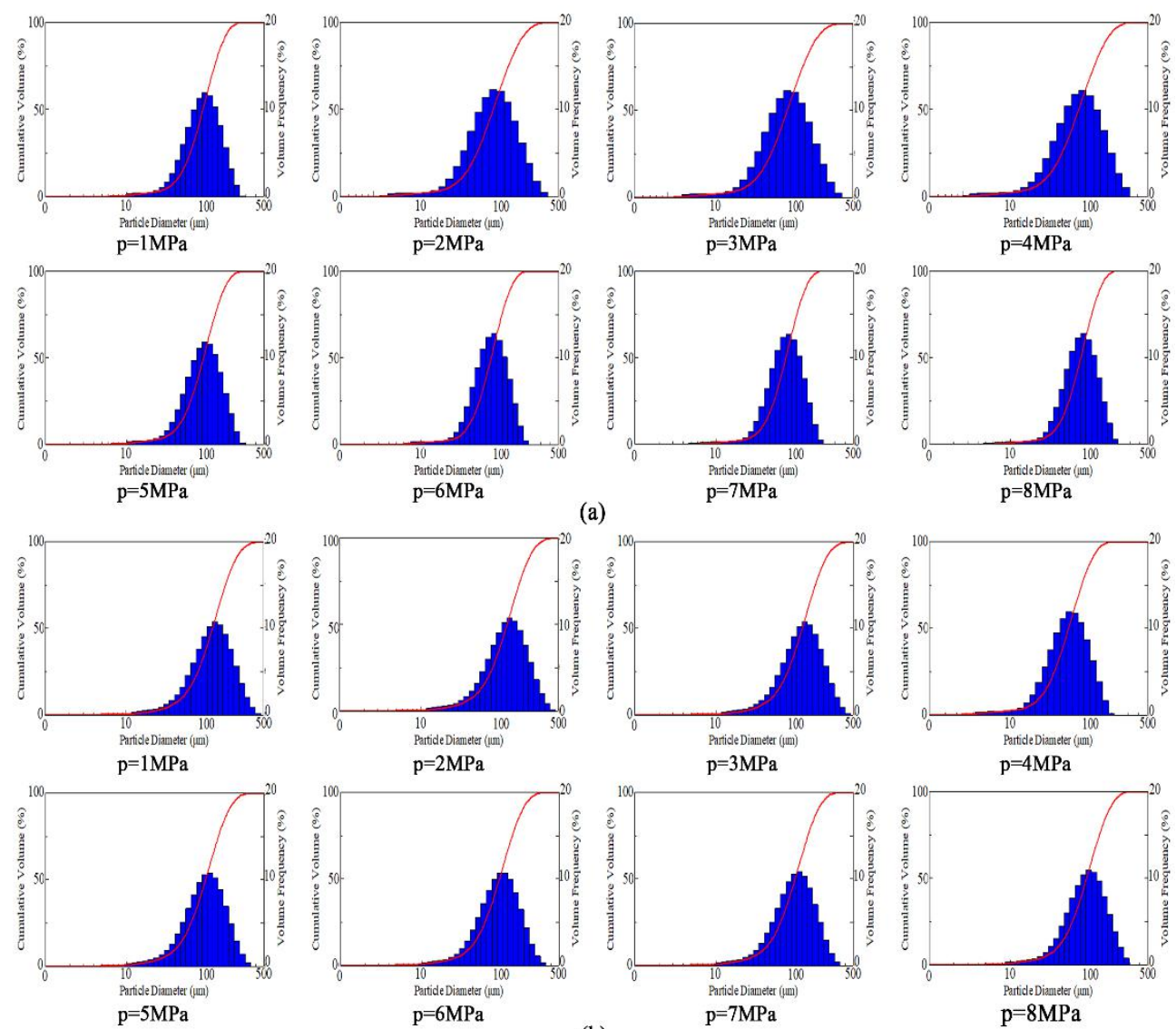

(b)

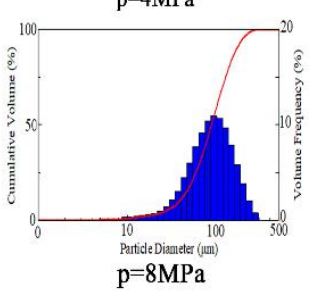

Fig. 8. Particle size of the two nozzles at each spray pressure, (a) spiral perforated nozzle, (b) spiral non-porous nozzle.

It can be seen from Fig. 7. and Fig. 8. that the droplet size of the spiral non-porous nozzle and the spiral perforated nozzle gradually decrease with the increase of the spray pressure, and the peak of the volume frequency of the two nozzles $\mathrm{DV}_{50}$ gradually goes to the left. That is, the direction in which the droplet size decreases is shifted. The droplet size of the spiral non-porous nozzle is always smaller than that of the spiral perforated nozzle at low pressure $(<5 \mathrm{MPa})$, and then the droplet size is gradually larger than that of the spiral perforated nozzle.

As the spray pressure is gradually increased, the internal flow rate of the nozzle is increased, and the higher the kinetic energy entering the inside of the nozzle, that is, the droplet is broken, and the effect of destroying the surface tension and the viscous force is better, so that the mist droplets sprayed from the nozzle The diameter gradually decreases as the spray pressure increases. When the droplets are ejected from the nozzle, secondary atomization occurs in a relatively close distance. Since the atomization angle of the spiral non-porous nozzle is large, the secondary atomization effect is better than that of the spiral aperture type. At the same time, since the spiral non-porous nozzle has no passage inside, the required work force to overcome the surface tension and viscous force of the droplet is 
smaller than that of the spiral-type nozzle, resulting in a spiral-type nozzle when the spray pressure is at a low pressure. The droplet size is smaller than that of the spiral non-porous nozzle. Therefore, in the actual working environment, if the spray pressure is required to be high, a spiral perforated nozzle can be selected.

\section{Conclusion}

Based on the experiment, this paper analyzes the variation of the droplet size during the spray dust reduction process of the two nozzles with different internal structures commonly used in the actual production process. At the same time, through the dust reduction experiment, the two nozzles selected are different. The dust reduction efficiency in the production environment, the relationship between the atomization characteristics and the dust reduction efficiency, and the comparison of the dust reduction efficiency at different spray pressures, summed up a nozzle model that is most suitable for actual production, thus reducing dust for the mining face. Provide theoretical guidance and specific experimental data, and ensure the dust reduction efficiency of underground coal mine work. The following main conclusions are drawn:

(1) The flow rates of both nozzles gradually increase as the spray pressure increases, and the flow coefficient of the spiral non-porous nozzle is small.

(2) The change of the atomization angle of the two nozzles first increases and then decreases with the increase of the spray pressure, and the atomization angle of the spiral non-porous nozzle is larger. At the same time, the range of the two nozzles gradually increases as the spray pressure increases, and the range of the spiral perforated nozzle is always larger than that of the spiral non-porous nozzle.

(3) When the spray pressure is gradually increased, the droplet size of the two nozzles selected in the experiment is gradually reduced, and the droplet size of the spiral perforated nozzle is always larger than that of the spiral non-porous nozzle before $5 \mathrm{MPa}$, and then gradually Become smaller. The main reason why the droplet size decreases with the increase of the spray pressure is that the increase of the spray pressure leads to an increase in the spray speed of the water droplets, so that the water droplets are completely split when they are ejected from the nozzle, resulting in a smaller droplet size.

\section{References}

[1] D. Chen, W.Nie, P. Cai, Z. Liu. Journal of Cleaner Production, The diffusion of dust in a fully-mechanized mining face with a mining height of $7 \mathrm{~m}$ and the application of wet dust-collecting nets. 205, 463-476 (2018)

[2] H. Li. Chinese Journal of Safety Science, Respirable dust in coal mine and its comprehensive control. 15, 67-69(2005)

[3] Gottesfeld, Nicas, Kephart, Balakrishnan, Rinehart. International Journal of Occupational and Environmental Health, Reduction of Respirable Silica Following the Introduction of Water Spray Applications in Indian Stone Crusher Mills. 14 (2008)

[4] G. Yang. Inner Mongolia Coal Economy, Research on Coal Mine Dust Prevention and Control. 16, 70+96 (2016)

[5] P.F. Wang, R.H. Liu, M. Tang, et al. Journal of Safety and Environment, Experimental study on the influence of nozzle diameter on dust reduction effect. 25, 114-120 (2015)

[6] L.H. LIu. Occupation and Health, Similarities and differences of coal mine dust concentration hygiene standards in workplace air at home and abroad. 31, 714-717 
(2015)

[7] J. Zuo, R. Rameezdeen, M. Hagger, et al. Journal of Cleaner Production, Dust pollution control on construction sites: Awareness and self-responsibility of managers. 166, 312-320 (2017)

[8] W. Nie, X. Ma, W. Cheng, et al. Process Safety and Environmental Protection, A novel spraying/negative-pressure secondary dust suppression device used in fully mechanized mining face: a case study. 103, 126-135 (2016)

[9] H. Yu, W. Cheng, H. Peng, et al. Advanced Powder Technology, An investigation of the nozzle's atomization dust suppression rules in a fully-mechanized excavation face based on the airflow-droplet-dust three-phase coupling model. 29, 941-956 (2018)

[10] Mei W. Tessum, Peter C. Raynor. Safety and Health at Work, Effects of Spray Surfactant and Particle Charge on Respirable Coal Dust Capture. 8, 296-305(2017)

[11] S. Xu, G. Fu. Coal Mine Safety, Analysis of Some Technical Problems in Coal Mine Dust Prevention and Control. 8, 37-39 (2000)

[12] G. Zhou. Research on spray dust reduction theory and technology in fully mechanized caving face. Shandong University of Science and Technology (2009)

[13] G. Zhou, W.M. Cheng, W. Nie, et al. Journal of Chongqing University, Theoretical analysis of the mechanism of high pressure spray jet atomization and water mist capture. 35, 121-126 (2012)

[14] S.P. Ma, Z.M. Zhai. Journal of Taiyuan University of Technology, Research and Analysis of Spray Dust Reduction Efficiency. 37, 327-330(2006)

[15] S.P. Ma, Z.M. Zhai. China Safety Science Journal, Study on Matching Efficiency of Spray Dust Reduction and Spray Parameters. 16, 84-89 (2016)

[16] Hadi Taghavifar, Mohammad Taghi Shervani-Tabar, Majid Abbasalizadeh. Applied Mathematical Modelling, Numerical study of the effects of injector needle movement and the nozzle irrigation angle on the internal fluid flow and spray structure of a group-hole nozzle layout. 39, 23-24 (2015)

[17] X.Q. Guan, J. Zhu, S.C. Nie, et al. Journal of Henan Chemical Industry, Experimental study on droplet distribution characteristics of spiral nozzles. 31, 30-32 (2014)

[18] C. Li, S.L. Wang, Z.S. Zhong, et al. Transactions of the Chinese Society of Agricultural Machinery, Jet Profile Geometric Model of Involute Atomizing Spiral Nozzle. 38, 75-78 (2007)

[19] G. Zhou, W.M. Cheng, W. Nie, et al. Journal of Chongqing University, Theoretical analysis of the mechanism of high pressure spray jet atomization and water mist capture. 35, 121-126(2012)

[20] H. Zhou, M.H. Fan, H.Y Yang, et al. Journal of Mechanical Engineering, Measurement of high-efficiency atomization characteristics of core nozzle. 40, 110-114(2004) 